\title{
Utilisation de la sédation consciente par inhalation de MEOPA en milieu hospitalier : étude statistique rétrospective sur 5 ans
}

\author{
Daniel Anastasio, Laurence Hein-Halbgewachs, Hélène Gautier, Cédric Seckinger, Eric Gérard \\ Service d'Odontologie, CHR Metz-Thionville, 1 rue du Friscaty, 57100 Thionville, France \\ d.anastasio@chr-metz-thionville.fr
}

Matériel et méthode : La population étudiée correspond aux patients traités sous sédation consciente par inhalation de MEOPA dans le service d'Odontologie de Thionville entre 2003 et 2008, soit 1165 sessions de soins.

L'analyse statistique a été réalisée à l'aide du logiciel SAS dans le Service d'Epidémiologie et d'Evaluation clinique du CHU de Nancy.

Résultats : Le sexe féminin représente $51,2 \%$ de l'effectif global, le sexe masculin représente 48,8\%. La classe d'âge la plus représentée est celle des 5-14 ans avec 51,8\% de l'effectif, la classe d'âge des $25-59$ ans représente $21,9 \%$, celle des $0-4$ ans $17,3 \%$, celle des $15-24$ ans $7,9 \%$, enfin celle des plus de 60 ans $1,1 \%$.

La catégorie majoritaire est celle des anxieux phobiques avec $60,1 \%$. Le groupe des jeunes enfants (de 5 ans) représente $17,1 \%$. Le groupe des déficients mentaux et cognitifs correspond à 19,2\% de l'effectif global. Enfin, les indications ponctuelles liées à l'acte sont de l'ordre de 3,6\%.

Le recrutement s'est fait majoritairement (58\%) par les chirurgiens dentistes libéraux et les médecins $(8,6 \%) ; 21,7 \%$ des patients ont été recrutés au sein du service. La distance entre le domicile et le centre d'investigation est de 0 à $4 \mathrm{~km}$ pour $40 \%$ des patients, de 5 à $10 \mathrm{~km}$ pour $8 \%$, de 11 à $30 \mathrm{~km}$ pour $34 \%$ et de plus de $30 \mathrm{~km}$ pour $16 \%$ de l'effectif.

Les avulsions dentaires représentent $32 \%$ des actes réalisés, les actes endodontiques sous anesthésie locale $37 \%$, les actes endodontiques sans anesthésie locale $13 \%$, les actes de chirurgie buccale 3,6\%. Le reste $(14,4 \%)$ regroupe les séances d'initiation, les détartrages et les actes de prothèse.

Dans plus de la moitié des sédations (53\%), la durée est comprise entre 10-19 mn, 15\% moins de 10 $\mathrm{mn}$ et $25 \%$ entre $20-29 \mathrm{mn}$. Les actes d'une durée de plus de $30 \mathrm{mn}$ représentent $7 \%$.

Les effets secondaires sont rares et concernent 5,8\% de l'effectif global. Les effets indésirables les plus fréquents sont les troubles digestifs $(60,3 \%)$, les troubles vagaux $(20,4 \%)$, les autres troubles représentent 19,4\% (malaise, toux, endormissement, hyperventilation, hyperexcitation, euphorie, hypoventilation). Aucun effet secondaire grave n'a été relevé.

L'évaluation du comportement a été réalisée à l'aide de l'échelle de Venham aux temps T0-X (premier contact), T0 (début de l'induction), T1 (fin de l'induction), T2 (temps de l'anesthésie) et T3 (temps d'exécution de l'acte). Les échecs sont rares et ne représentent que 4,1\%, soit un total de 48 échecs. Les échecs de sédation sont au nombre de 43 cas (3,7\%), les échecs de sédation et de 1'acte sont au nombre de 5 cas $(0,4 \%)$.

Discussion : Cette étude montre la pertinence de cette technique. Les résultats sont similaires à ceux d'autres études françaises (Hennequin et al. 2004 sur 1205 sessions de soins, San Fulgencio et al. 2004 sur 344 sessions de soins).

Cette étude a été réalisée dans un Service d'Odontologie polyvalente. Elle offre donc une vision des bénéfices de la sédation par inhalation dans une population composée de tous types de patients nécessitant cette prise en charge spécifique. Cependant, pour affirmer statistiquement l'intérêt de cette technique, il aurait fallu comparer 2 groupes sélectionnés de manière aléatoire, l'un ayant bénéficié d'une sédation pour les soins et l'autre n'ayant pas reçu cette technique pour les soins. 